\title{
线粒体靶向过氧化氢荧光探针的研究进展
}

\author{
李娇娇班立夫汤立军* \\ (渤海大学化学化工学院 辽宁锦州 121013)
}

\begin{abstract}
摘要 控制线粒体过氧化氢的含量可以为细胞存活、生长、分化和维持发挥有益的作用. 然而, 线粒体过氧化氢的异 常产生可能会破坏生物大分子以及细胞的结构, 导致老化、突变甚至癌症的发生. 因此, 迫切需要能够在活细胞中特别 是在线粒体中对过氧化氢的水平变化进行有效监测的手段. 为此, 开发、设计了多种监测线粒体中过氧化氢水平变化 的苂光探针. 以靶向基团结构为分类依据，总结了近年来线粒体靶向过氧化氢荧光探针的研究进展，介绍了三苯基膦 鎓盐类探针、吡啶阳离子类探针、喹啉阳离子类探针、吲哚阳离子类探针和其他阳离子型探针，总结了这些探针的结 构、荧光行为及生物成像等. 这些荧光探针将成为疾病诊断和病理研究过程中有力的分子工具.
\end{abstract}

关键词 过氧化氢; 线粒体; 荧光探针; 正电荷基团; 传感机理

\section{Research Progress in Mitochondrial Targeting Fluorescent Probes for Hydrogen Peroxide}

\author{
Li, Jiaojiao \\ Ban, Lifu \\ Tang, Lijun* \\ (College of Chemistry and Chemical Engineering, Bohai University, Jinzhou, Liaoning 121013)
}

\begin{abstract}
Controlling the content of mitochondrial hydrogen peroxide can play a beneficial role in cell survival, growth, differentiation and maintenance. However, the abnormal production of mitochondrial hydrogen peroxide may destroy the structure of biological macromolecules and cells, and promote the occurrence of aging, mutation and even cancer. Therefore, there is an urgent need for means to effectively monitor changes in hydrogen peroxide levels in living cells, especially in mitochondria. To this end, a variety of fluorescent probes have been developed and designed to monitor changes in hydrogen peroxide levels in mitochondria. The recent progress in mitochondrial targeting fluorescent probes for hydrogen peroxide based on the structure of targeting groups is summarized, and triphenylphosphonium probes, pyridinium probes, quinolinium probes, indolium probes, and other cationic probes are introduced, including the structure, fluorescence behavior and biological imaging of these probes. These fluorescent probes will become powerful molecular tools in future disease diagnosis and pathological research.
\end{abstract}

Keywords hydrogen peroxide; mitochondria; fluorescent probe; positively charged group; sensing mechanism

过氧化氢, 是活性氧(ROS)家族的重要成员之一, 影响着生物的生长、发育和适应能力 ${ }^{[1-3]}$. 在生理水平上, 过氧化氢能够调节生物活性, 被公认是衰老和疾病中氧 化应激的标志物 ${ }^{[1]}$, 是病原体入侵时免疫反应的防御 剂 $^{[4]}$, 也是与细胞增殖、迁移和分化有关的信使分子 ${ }^{[5-6]}$. 在活细胞中, 过氧化氢的释放和分隔受到精确地控制和 精细地调节. 然而, 在外源性刺激下过氧化氢的异常产 生可能会攻击细胞生物分子 ${ }^{[7]}$. 大量证据表明, 过氧化 氢的过量产生和积累可能会导致各种人类疾病, 如心血
管疾病、糖尿病、帕金森氏病、阿尔茨海默病、亨廷顿 氏病、代谢性疾病和癌症等 ${ }^{[8-9]}$.

近年来，报道了许多检测过氧化氢的方法，如分光 光度法、电化学分析法、色谱法、苂光光谱法和化学发 光法等 ${ }^{[10]}$. 然而, 由于生物环境的复杂性,一些方法会 导致组织损伤, 影响体内检测. 苂光标记是一种强大的 技术，具有无干扰、实时和可视化等特点 ${ }^{[11-13]}$. 其中，荧 光探针作为一种重要的工具, 与苂光蛋白、量子点和纳 米粒子等其他苂光指示剂相比, 具有很强的灵敏度和选

\footnotetext{
* Corresponding author. E-mail: ljtang@bhu.edu.cn

Received June 12, 2020; revised July 8, 2020; published online July 30, 2020.

Project supported by the National Natural Science Foundation of China (Nos. 21878023, U1608222) and the Program for Distinguished Professor of Liaoning Province.
}

国家自然科学基金(Nos. 21878023, U1608222)和辽宁省特聘教授支持计划资助项目. 
择性、应用方便和成本低等优点, 受到化学家和生物学 家的普遍青睐 ${ }^{[14-19]}$. 因此, 开发过氧化氢荧光探针对人 体健康和临床诊断都具有重要意义.

近年来, 荧光探针的设计取得了重大进展, 许多探 针被用于生物系统中过氧化氢的成像. 但是, 大多数过 氧化氢探针通过自由扩散进入细胞, 随机分布在细胞质 中, 缺乏细胞器靶向或积累的能力. 然而, 在细胞器水 平上, 线粒体是过氧化氢的主要来源, 也是氧消耗的主 要细胞器. 数据表明, 线粒体过氧化氢有助于细胞的存 活、生长、分化和维持 ${ }^{[20-21]}$. 实现线粒体中过氧化氢的 可视化检测对于某些疾病的预防、诊断以及病理的研究 具有十分重要的意义 ${ }^{[22-24]}$. 考虑到上述要求, 研究者们 设计开发了一些能特异性识别过氧化氢并且靶向定位 于线粒体的苂光探针.

根据现有文献报道，能够特异性定位于线粒体的苂 光探针多数基于带有正电荷的基团, 从而对线粒体进行 靶向定位 ${ }^{[25-26]}$. 探针在线粒体中的积累可以通过膜电 位、阳离子的心磷脂络合或弱酸的离子捕获来驱动 ${ }^{[27]}$. 具体而言, 线粒体膜的电位通常为 $-180 \mathrm{mV}$, 因此带有 阳离子的染料可以轻松地穿透线粒体膜, 随后在其内部 进行积累 ${ }^{[28]}$. 三苯基膦鈆盐、吲哚鎓盐、季铵化吡啶基、 季铵化喹啉基和氧鎓离子等带正电荷的基团, 不仅能提 高探针的水溶性, 还可以使探针在活细胞中展现出优异 的线粒体靶向性 ${ }^{[24,29]}$.

本文将总结已报道的能特异性识别过氧化氢并且 靶向定位于线粒体的苂光探针. 根据不同的线粒体靶向 定位基团, 将探针分类为: 三苯基膦鎓盐类探针、吡啶 阳离子类探针、喹啉阳离子类探针、吲哚阳离子类探针 以及其他阳离子型探针. 对这些探针进行了总结, 阐述 了我们对活细胞中线粒体过氧化氢的可视化监测方面 的观点.

\section{1 三苯基膦鍮盐类探针}

探针在线粒体内数百倍的积累是由膜电位驱动的, 带有三苯基膦(TPP)标记的化合物可以选择性地聚集在 线粒体 ${ }^{[30-32]}$.

2008 年, Chang 等 ${ }^{[33]}$ 以罗丹明染料为苂光团, 三苯 基膦鈆盐为线粒体靶向基团, 设计合成了第一个线粒体 靶向过氧化氢苂光探针 1 . 由于具有化学特异性的嗍酸 酯开关, 探针 1 对过氧化氢的选择性优于其他活性氧物 种 (如超氧化物、一氧化氮和羟基自由基等). 与对照细 胞相比, 在负载了探针 $\mathbf{1}$ 的 HeLa 细胞中加入 $100 \mu \mathrm{mol} /$ $\mathrm{L}$ 的过氧化氢, 苂光信号明显增强 $\left(\lambda_{\mathrm{em}}=528 \mathrm{~nm}\right)$, 说明 探针 1 能检测活细胞内过氧化氢. 使用探针 $\mathbf{1}$ 与商品化 的线粒体指示剂 MitoTracker Deep Red 进行共染色实验,
证实了探针被保留在线粒体中. 此外, 在非洲绿猴肾细 胞(Cos-7)、人胚胎肾细胞 293 (HEK293)和中国仓鼠卵巢 细胞(CHO.K1)细胞中进行的类似实验也得到了相似的 结果, 扩大了探针的使用范围. 数据表明, 探针 $\mathbf{1}$ 能够 对各种哺乳动物细胞内线粒体过氧化氢的水平变化进 行成像.



2012 年, Kim 课题组 ${ }^{[34]}$ 开发了第一个双光子比率过 氧化氢探针 2, 它可以通过双光子显微镜(TPM)检测活 细胞和完整组织中的过氧化氢. 该探针以 6-(苯并 $[d]$ 噻 唑-2'-基)-2-( $N, N$-二甲基氨基)菜作为报告基, 以基于嗍 酸酯的氨基甲酸酯离去基团作为过氧化氢的响应位点, 以三苯基膦鎓盐作为线粒体靶向位点. 经过修饰，硼酸 酯和 TPP 在分子两端, 以最大程度减少它们之间的相互 作用. 与过氧化氢反应后, 探针 2 在 3-(N-吗啉基)丙磺 酸(MOPS)缓冲液中的荧光峰从 $470 \mathrm{~nm}$ 变为 $545 \mathrm{~nm}$, 溶 液的颜色由蓝色变为黄色. 此外, $400 \sim 470 \mathrm{~nm}\left(F_{\text {blue }}\right)$ 和 $530 \sim 600 \mathrm{~nm}\left(F_{\text {yellow }}\right)$ 的发射强度之比增加了 75 倍. 该 探针灵敏度高, 检测限为 $4.6 \mu \mathrm{mol} / \mathrm{L}$, 对过氧化氢具有 较高的选择性. 使用探针 2 和线粒体指示剂 MitoTracker Red 在 Raw 264.7 小鼠巨噬细胞进行了共定位实验，皮 尔森共定位系数为 0.91 , 表明探针主要存在线粒体中. 该探针可以通过 TPM 观察到 $1 \times 10^{-4} \sim 1.8 \times 10^{-4} \mathrm{~m}$ 深 度的活细胞和活组织中线粒体过氧化氢水平的变化.

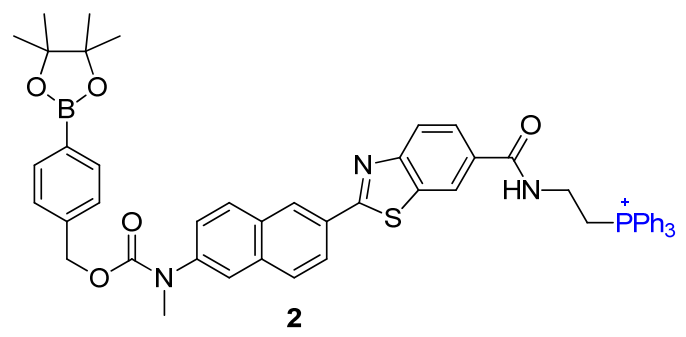

2014 年, 吴水珠课题组 ${ }^{[35]}$ 开发了第一个用于检测 线粒体过氧化氢的多功能荧光纳米探针 3. 该纳米探针 的设计是以碳点 (Carbon dot, CD) 作为传感系统的载体, 将过氧化氢的识别元件(PFI)共价连接到碳点上, 并将 TPP 整合到碳点上以靶向活细胞中线粒体. CD 上的 PFI 为传感系统提供了一种基于荧光共振能量转移(FRET) 机制的过氧化氢比率探针. 使用线粒体特异性染料(罗 
丹明 123)与菼光纳米探针 3 共同染色 L929 和 Raw 264.7 细胞，共聚焦荧光图像表明，探针表面具有靶向配体时 能特异性染色活细胞中线粒体区域. 探针 3 可以检测 L929 细胞中的外源性过氧化氢以及佛波醇肉豆冦乙酸 酯(PMA)刺激下 Raw 264.7 细胞中线粒体内产生的过氧 化氢. 由于具有 FRET 机制, 该纳米探针可以在单波长 激发下产生双色发射, 与仅具有单一发射或需要多个激 发波长的多色系统相比, 提供了更好的、更便捷的比率 检测方法. 探针 3 还具有高水溶性、令人满意的细胞通 透性、非常低的细胞毒性、基于 FRET 的比率传感和线 粒体靶向能力等优点. 唐波课题组 ${ }^{[36]}$ 也开发了一种线 粒体靶向苂光纳米传感器, 用于实时检测活细胞中过氧 化氢和 $\mathrm{pH}$ 的波动. 在小尺寸介孔二氧化硅纳米粒子 (MSN)中负载了检测 $\mathrm{pH}$ 和过氧化氢的苂光探针, 然后 将聚乙烯亚胺附着在 MSN 的孔上，再通过三苯基膦鎓 盐进一步修饰，使其靶向活细胞中的线粒体.

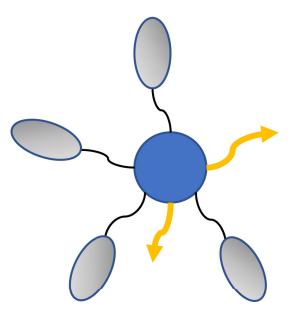

3

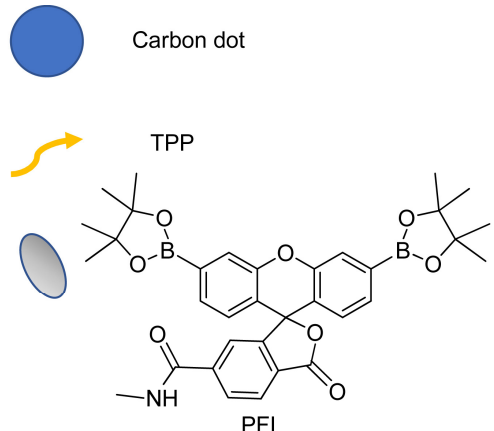

2016 年, 肖瑜峰 ${ }^{[37]}$ 报道了一种线粒体靶向过氧化 氢探针 4, 该探针以硫磺菊素衍生物作为苂光团, 三苯 基膦鎓盐作为线粒体靶向位点. 探针 4 中带有一个特异 性响应过氧化氢的硼酸酯开关, 在过氧化氢的氧化下, 首先在硼原子上形成氢键, 然后经过迁移、重排和水解 生成羟基化合物(Scheme 1). 探针 4 可以通过苂光增强 来检测过氧化氢. 在共染色实验中, 探针 4 和线粒体特 异性苂光染料(MitoTracker Deep Red)的苂光图显示出 良好的重叠, 说明探针 4 可高效定位于线粒体. 此外, 探针 4 不仅能用于检测外源性过氧化氢, 还能用于氧化 应激引发的内源性过氧化氢的检测.

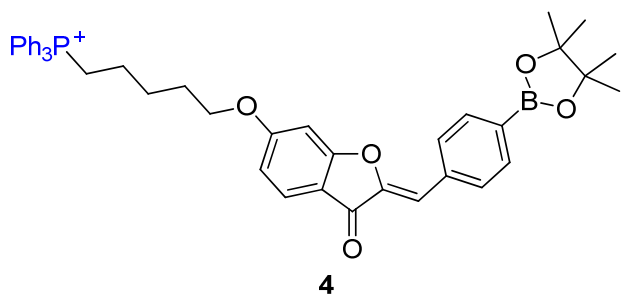

2019 年, 赵一兵和吴川六课题组 ${ }^{[38]}$ 提出了一种通 用且可靠的双猝灭概念, 用于设计低背景苂光的苂光探

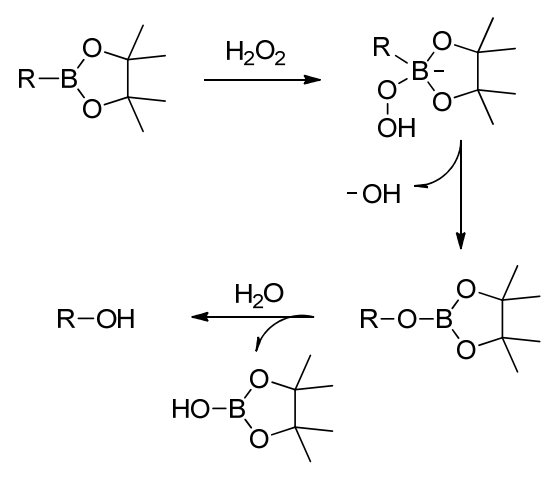

图式 1 基于嗍酸酯的苂光探针与 $\mathrm{H}_{2} \mathrm{O}_{2}$ 的反应机理 Scheme 1 Reaction mechanism of boronate-based fluorescent probe with $\mathrm{H}_{2} \mathrm{O}_{2}$

针, 并开发了一种用于检测癌细胞中线粒体内源性过氧 化氢的苂光探针 5. 选择 $\mathrm{KB}$ 细胞(一种表皮样口腔癌异 倍体细胞系)作为模型癌细胞与探针 $\mathbf{5}$ 进行研究, 细胞 的荧光成像表明该探针可以快速响应癌细胞中内源性 过氧化氢. 在 $\mathrm{KB}$ 细胞中进行共定位实验，探针 5 和线 粒体特异性苂光染料 MitoTracker Red 的共聚焦荧光图 像表明探针能定位于线粒体. 此外, 探针 5 还能监测不 同温度下过氧化氢的变化, 突显了其对复杂生物系统中 内源性过氧化氢的检测具有较高灵敏度和特异性的特 点. 该探针同时利用硼酸酯驱动的内酰胺的形成和可消 除的猝灭单元(即双猝灭效应)来减少背景苂光的干扰， 最终导致了大于 50 倍的荧光增强 $\left(\lambda_{\mathrm{ex}}=470 \mathrm{~nm}, \lambda_{\mathrm{em}}=\right.$ $528 \mathrm{~nm})$.

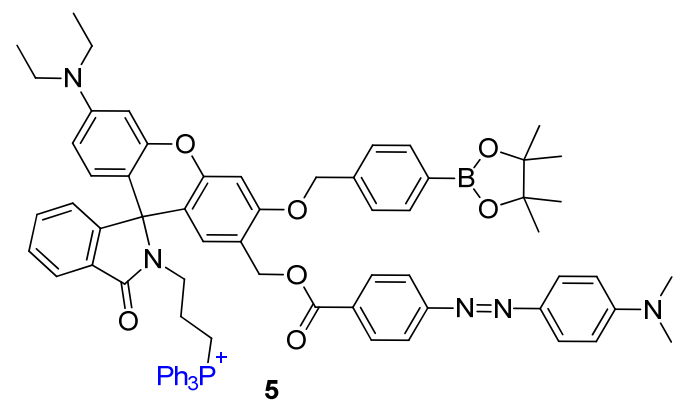

由于三苯基膦阳离子具有亲脂性，能迅速通过生物 膜定位于线粒体，具有优异的线粒体靶向性，所以被引 入到许多探针和苂光纳米传感器中作为线粒体的靶向 基团，但是这类探针还存在一些问题. 例如，这类小分 子探针的发射波长通常在可见光区域，缺乏更深的组织 穿透力, 容易受到背景苂光的干扰. 大多数过氧化氢探 针应用于生物细胞中, 探针的细胞毒性是在细胞内检测 时使用探针的关键参数. 然而, 在生物应用中三苯基膦 因为毒性受到限制 ${ }^{[39]}$. 因此, 研究者相继开发了其他种 类的线粒体靶向基团. 


\section{2 吡啶阳离子类探针}

吡啶阳离子作为线粒体靶向基团具有优越的膜通 透性, 能使探针在短时间内迅速进入活细胞线粒体 中 $^{[40]}$.

2016 年，林伟英课题组 ${ }^{[41]}$ 设计合成了第一个可检 测线粒体粘度和过氧化氢的双响应型苂光探针 6 . 在探 针中, 双键和苯环用作供电子基团 ( N,N -二甲基氨基)和 吸电子基团(吡啶阳离子)的连接基, 可以使分子在激发 态下自由旋转. 由于探针 6 具有扭曲的分子内电荷转移 (TICT)机制, 它可以分别对粘度和过氧化氢进行响应, 并伴随着大的“开启” 型苂光信号, 发射波长分别为 604 和 $510 \mathrm{~nm}$. 探针 6 对过氧化氢的选择性高于其它活性 氧、活性氮及活性硫, 并且具有较高的灵敏度. 共定位 实验结果表明, 探针 6 与线粒体定位染料 Mito Tracker Deep Red 的染色结果吻合较好, 探针通道和 MitoTracker Deep Red 通道的强度散点图具有较高的相 关性, 皮尔森共定位系数为 0.93 . 细胞成像证实探针 6 具有膜通透性, 可用于检测线粒体过氧化氢的水平和粘 度的变化.

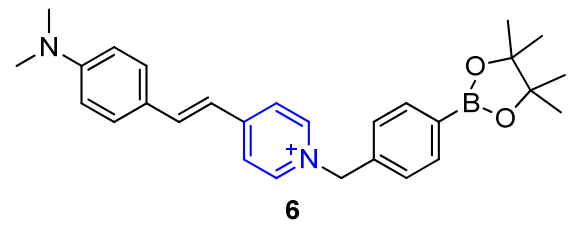

2017 年，林伟英等 ${ }^{[42]}$ 设计合成了一种具有大斯托 克斯位移的线粒体靶向苂光探针 7 , 可以用于水溶液和 活细胞中过氧化氢的检测. 探针 7 以咔唑基衍生物作为 苂光团，吡啶鎓盐和硼酸酯单元分别作为线粒体靶向基 才和过氧化氢的识别位点. 与过氧化氢反应前, 大多数 含有硼酸酯单元的过氧化氢探针具有较低的苂光量子 效率, 这是由于苂光通过分子内激发的光诱导电子转移 (PET)过程猝灭所致, 与过氧化氢反应后荧光团会呈现 强苂光. 但是与大多数含有硼酸酯单元的探针不同, 随 着过氧化氢的加入, 探针 7 的苂光强度明显降低, 表现 出 “关断” 响应 $\left(\lambda_{\mathrm{ex}}=405 \mathrm{~nm}, \lambda_{\mathrm{em}}=575 \mathrm{~nm}\right)$. 探针 7 可 以通过菼光猝灭在磷酸缓冲盐溶液 $(\mathrm{PBS})$ 中高灵敏度 $($ 检 测限为 $2.8 \mu \mathrm{mol} / \mathrm{L}$ ) 和高选择性地检测过氧化氢. 该探针 在各种溶液中都显示出较大的斯托克斯位移(如在水中 为 $157 \mathrm{~nm}$ ). 将探针 7 和商品化线粒体染料 MitoTracker Red 在 RAW 264.7 细胞中进行共定位实验, 皮尔森共定 位系数为 0.90 , 表明探针 7 主要位于线粒体中. 苂光成 像表明，该探针适合检测活体 RAW 264.7 细胞线粒体中 外源性和内源性过氧化氢.



2018 年, 我们课题组 ${ }^{[43]}$ 设计合成了一种线粒体靶 向比率型荧光探针 8 , 该探针具有近红外发射和很大的 斯托克斯位移 $(357 \mathrm{~nm})$. 探针 8 以苯硼酸酯作为过氧化 氢的响应基团和激发态分子内质子转移(ESIPT)机制的 阻断基团. 核磁共振氢谱和质谱分析结果表明，加入过 氧化氢后探针 8 将依次进行氧化一水解和 1,6-消除反应, 释放出酚羟基并恢复苂光团的 ESIPT 机制. 通过苂光光 谱中观察到, 加入过氧化氢的探针 8 在 $669 \mathrm{~nm}$ 处出现新 的发射带. 此外，该探针对过氧化氢的选择性高于其他 活性氧/氮(ROS/RNS)、生物硫醇和阴离子，检测限低至 $5.9 \times 10^{-7} \mathrm{~mol} / \mathrm{L}$. 在人非小细胞肺癌细胞(A549)中进行 探针 8 和 Mito- Tracker Green 的共定位实验，两个通道 的苂光信号重合度非常好，皮尔森共定位系数达 0.948 , 验证了探针 8 的线粒体靶向能力. 此外, 探针 $\mathbf{8}$ 可用于 活体 A549 细胞中的外源性和内源性过氧化氢的检测.

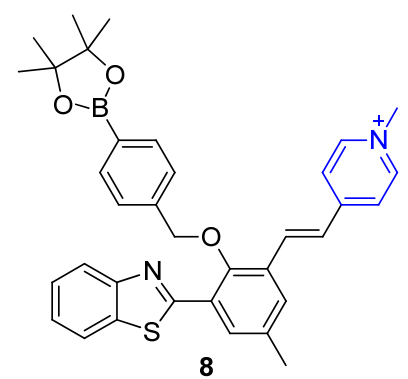

2018 年, 葛剑锋课题组 ${ }^{[44]}$ 开发了一种具有吡喃一吡 啶骨架的苂光探针 9. 与过氧化氢作用前, 由于分子内 PET 过程，探针 9 几乎没有苂光. 加入过氧化氢后引发 苯基硼酸酯基团的自脱落, 释放出中性吡啶结构, 分子 内 PET 机制消失，在 $604 \mathrm{~nm}$ 处表现出明显的苂光 “开 启” 响应(苂光增强 33 倍), 溶液由无色变为鲜红色. 用 探针 9 在 $\mathrm{HeLa}$ 细胞和人胃黏膜上皮细胞(Ges-1)细胞中 进行共聚焦荧光成像，其发射与 Mito-Tracker Green 苂 光匹配良好，表明该探针可用于检测线粒体过氧化氢.

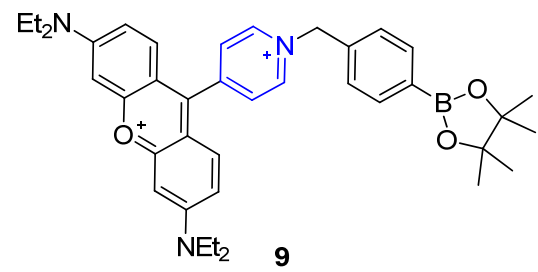


2019 年, 李林等 ${ }^{[45]}$ 设计合成了一种新型双光子(TP) 荧光探针 10, 它可以使用两个不同的通道同时监测线 粒体粘度和过氧化氢水平的变化. 探针 $\mathbf{1 0}$ 以 $N, N$-二甲 氨基作为强电子供体部分, 与作为吸电子部分的吡啶阳 离子结合, 形成了 “电子供体- $\pi$-电子受体 $(\mathrm{D}-\pi-\mathrm{A})$ ” 分子 构型. 在低粘度介质中, 由于探针 $\mathbf{1 0}$ 在激发态存在 TICT 过程而无荧光, 随着介质粘度的增加, TICT 过程 受到抑制, 探针 10 发出强烈的近红外苂光 $\left(\lambda_{\mathrm{em}}=730\right.$ $\mathrm{nm})$. 探针与过氧化氢作用后, TICT 过程消失, 可以在 $585 \mathrm{~nm}$ 处检测到明显的苂光信号. 探针 $\mathbf{1 0}$ 与线粒体染 料 MitoTracker Red 进行共染色实验, 皮尔森共定位系 数为 0.81 , 表明探针 $\mathbf{1 0}$ 主要位于线粒体中. 探针 $\mathbf{1 0}$ 可 用于活体系统(包括活细胞、斑马鱼和果蝇)的成像, 并 可以通过单光子和双光子苂光共聚焦显微镜监测线粒 体中粘度和过氧化氢水平的变化.



2020 年, 田阳课题组 ${ }^{[46]}$ 合成了一个双光子苂光寿 命探针 11, 该探针可以通过不同的荧光信号同时监测 线粒体中过氧化氢和 $5^{\prime}$-三磷酸腺苷(ATP)的水平变化. 与过氧化氢反应后, 探针 $\mathbf{1 1}$ 在 $470 \mathrm{~nm}$ 处发射强度显著 增加; 随着 ATP 的加入, 探针 11 在 $590 \mathrm{~nm}$ 处出现了一 个新的发射峰. 探针 11 在过氧化氢 $(0.4 \sim 10 \mu \mathrm{mol} / \mathrm{L})$ 和 ATP $(0.5 \sim 15 \mathrm{mmol} / \mathrm{L})$ 的检测范围内表现出较高的选择 性和准确性. 过氧化氢通道与线粒体染料 MitoTracker Red 通道的荧光信号吻合良好(皮尔森共定位系数为 0.95). 来自 ATP 通道的苂光信号与 MitoTracker Red 通 道的荧光信号也有很好的重叠 (皮尔森共定位系数为 0.96), 表明了探针 11 可以有效地进入线粒体. 使用探针 11 可以通过不同的荧光信号测定过氧化氢和 ATP 的水 平, 并且没有 FRET 和串扰. 利用探针 $\mathbf{1 1}$ 还可以准确地

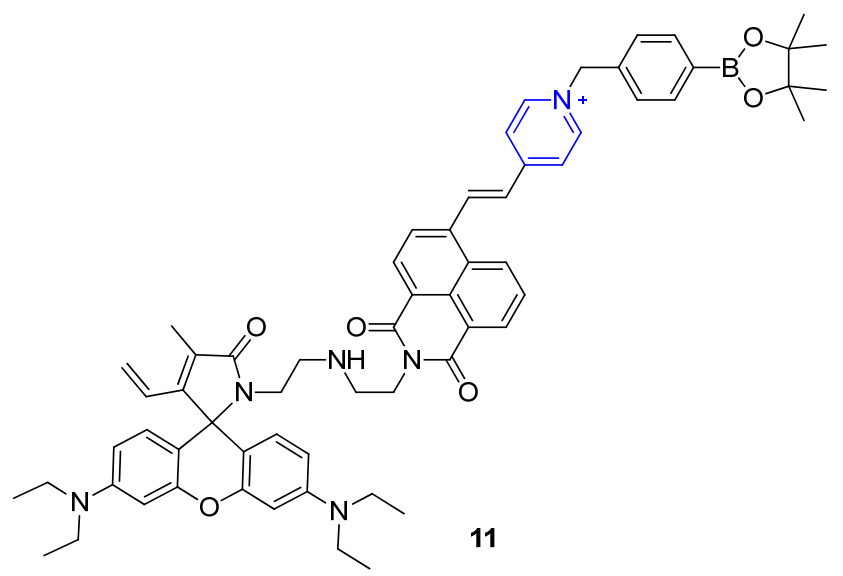

研究过氧化氢与 ATP 的关系, 有助于理解线粒体过氧化 氢和 ATP 在参与大脑的生理和病理过程中的作用.

目前报道的以季铵化吡啶作为定位基团的线粒体 靶向过氧化氢探针, 具有良好的线粒体靶向能力. 由于 其响应机制(如 TICT、PET、ESIPT 等), 许多此类探针 具有近红外发射和较大的斯托克斯位移, 有利于生物应 用及荧光成像研究. 基于 TICT 机制设计的双功能荧光 探针，实现了以不同通道同时监测线粒体中粘度和过氧 化氢的水平, 为今后过氧化氢探针和粘度探针的设计提 供了新思路. 然而, 基于分子内电荷转移(ICT)、PET 等 机制的探针可能存在荧光量子产率较低的问题, 需要进 一步完善.

\section{3 喹啉阳离子类探针}

2016 年, 邵士俊等 ${ }^{[39]}$ 开发了一种靶向定位于线粒 体的阳离子探针 12. 该探针采用咔唑基团作为荧光团, 以对甲基苯硼酸频哪醇酯作为过氧化氢的反应基团, 季 铵化喹啉单元作为线粒体靶向基团. 探针 12 由于 PET 效应表现出微弱的苂光. 与过氧化氢反应后 PET 效应消 失，释放出咔唑衍生物的苂光，导致 “开启” 型荧光响 应. 加入过氧化氢后, 探针 12 在 $527 \mathrm{~nm}$ 处的苂光强度 增加了 33 倍, 溶液的颜色由浅红色变为无色. 探针 $\mathbf{1 2}$ 具有优异的选择性, 仅对过氧化氢表现出显著的荧光增 强现象. 在活细胞中进行共定位实验, 探针 12 与 Mito Tracker Deep Red 的苂光信号非常吻合，证实了探针主 要存在于线粒体中. 该探针能对活细胞中内源性和外源 性过氧化氢的水平变化作出响应, 并已被成功应用于 $\mathrm{HeLa}$ 细胞中内源性过氧化氢的成像.

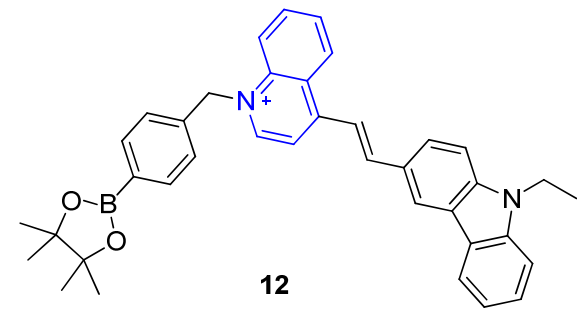

2018 年, 宋相志课题组 ${ }^{[29]}$ 利用 ICT 与 ESIPT 机制 的结合, 合成了一种用于检测过氧化氢的比率型荧光探 针 13. 探针 13 是具有近红外发射和大斯托克斯位移 (102 nm)的 2-(2'-羟基苯基)苯并噻唑(HBT)衍生物, 它以 对甲基苯硼酸频哪醇酯和喹啉季铵盐作为过氧化氢和 线粒体的活性位点. 探针 13 与过氧化氢反应, 限制了 ICT 效应，导致发射峰从 $666 \mathrm{~nm}$ 变为 $594 \mathrm{~nm}$, 斯托克斯 位移从 $102 \mathrm{~nm}$ 变为 $254 \mathrm{~nm}$. 通过共聚焦成像实验, 证 实了探针 13 能检测活细胞中内源性和外源性过氧化氢. 使用罗丹明 123 与探针 13 在 HeLa 细胞中进行共定位实 
验, 结果表明探针可以特异性染色线粒体. 与其它基于 苯并噻唑衍生物的比率探针不同, 探针 13 与过氧化氢 反应后不仅保留了分子内 ESIPT 过程, 还保持了大的斯 托克斯位移 $(254 \mathrm{~nm})$.

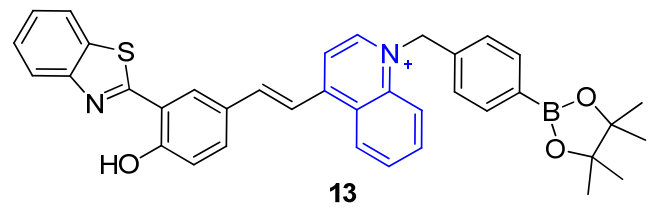

2020 年, 刘志洪课题组 ${ }^{[47]}$ 报道了一种具有大斯托 克斯位移的近红外发射荧光探针 14, 用于检测阿尔兹 海默症活鼠大脑中的线粒体粘度和过氧化氢. 探针 14 中亲脂性的 $\pi$-共轭噻吩桥不仅可以使探针扩展到近红 外发射, 提高血-脑屏障(BBB)的穿透性, 还能作为供电 子基团 ( N,N -二甲氨基)和吸电子基团(喹啉阳离子)的连 接基. 该探针对过氧化氢的检测有较高的灵敏度, 700 $\mathrm{nm}$ 处的苂光强度随着过氧化氢浓度的增加而增强, 呈 现 “开启” 型荧光响应. 同时, 随着粘度逐步增加, 探针 在 $800 \mathrm{~nm}$ 处的苂光强度逐渐增强. 这种与粘度相关的 荧光变化清楚地验证了传感系统中存在典型的 TICT 过 程. 探针 14 对目标物的检测具有较高的灵敏度、选择性 和较大的斯托克斯位移(过氧化氢: $260 \mathrm{~nm}$, 粘度: 230 $\mathrm{nm})$. 在 HeLa 细胞中进行了共定位实验, 其较大的皮尔 森共定位系数(过氧化氢: 0.957 , 粘度: 0.968)证实了探针 14 在活细胞中分别响应线粒体过氧化氢和粘度的能力.

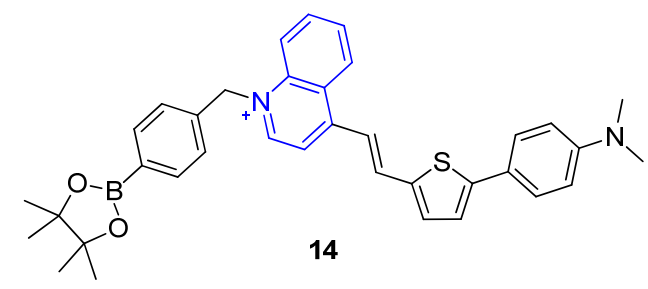

由于含喹啉阳离子的过氧化氢荧光探针与含吡啶 阳离子的过氧化氢探针的响应机制相似, 大多数喹啉阳 离子型探针具有大的斯托克斯位移, 有利于克服 “自吸 收” 效应. 部分研究通过扩展探针分子的 $\pi$ 共轭体系长 度实现近红外发射, 以提高组织穿透性, 降低背景荧光 干扰. 但是, 和吡啶阳离子类探针一样, 部分喹啉阳离 子探针也具有荧光量子产率较低的问题.

\section{4 吲哚阳离子类探针}

2016 年, 唐波课题组 ${ }^{[88]}$ 基于硼酸脱保护的机制合 成了线粒体过氧化氢探针 15 . 该探针以嗍酸作为过氧 化氢的识别基团和 ICT 过程的掩蔽基团. 探针 15 与过 氧化氢反应后, 嗍酸基团转化为氧负离子, 形成强烈的
推-拉共轭体系, 发生 ICT 效应, 并展现出明显的荧光 增强现象 $\left(\lambda_{\mathrm{em}}=555 \mathrm{~nm}\right)$. 探针 15 对过氧化氢的检测具 有较高的灵敏度和选择性以及出色的光稳定性. 在 HepG2 细胞(来源于肝癌组织)和小鼠乳腺癌细胞(4T1) 中进行共染色实验，探针 $\mathbf{1 5}$ 与 Mito-Tracker Deep Red 的苂光信号吻合良好(重叠系数 0.90), 验证了探针 15 线 粒体靶向的能力. 同时, 作者合成了另一个探针, 用于 监测内质网中过氧化氢的水平变化. 利用这两种探针同 时对过氧化氢水平进行苂光成像, 发现在各种刺激诱导 的细胞调亡过程中, 线粒体和内质网中过氧化氢的变化 是不同的. 这是细胞调亡过程中不同细胞器中过氧化氢 水平同时荧光成像的第一个例子.

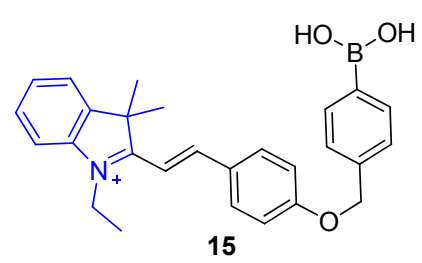

同年，唐波等 ${ }^{[49]}$ 又开发了一个线粒体靶向近红外 发射的苂光探针 16, 它利用了一个独特的响应开关 $(\alpha-$ 酮酰胺基), 对过氧化氢的检测具有较高的灵敏度和良 好的选择性. 探针 16 是一个受 ICT 机制调节的 “关闭” 型荧光探针. 加入过氧化氢后, 荧光团 $\mathbf{C y}-\mathbf{N H}_{2}$ 被释放 (Eq. 1)，并且由于 ICT 机制表现出明显的荧光增强现象. 荧光光谱中, $704 \mathrm{~nm}$ 处的荧光强度随着过氧化氢浓度的 增加而逐渐增加. 在 HepG2 细胞中探针 16 的苂光信号 与线粒体追踪器 Mito-Tracker Green 的苂光信号吻合良 好, 皮尔森共定位系数为 0.96 , 表明探针 16 特异性存在

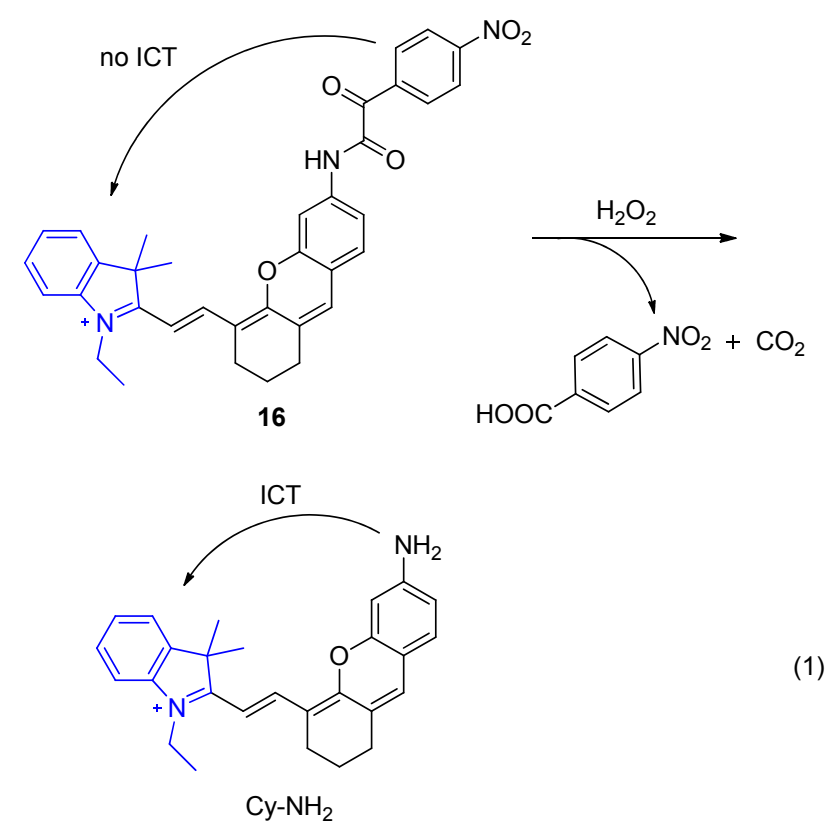


于线粒体. 使用探针 16 可以实现缺血再灌注损伤模型 中过氧化氢水平的可视化.

2019 年, 陈令新等 ${ }^{[50]}$ 开发并合成了一个比率型苂 光探针 17 , 它对过氧化氢的检测具有良好的选择性和 较高的灵敏度(检测限低至 $20 \mu \mathrm{mol} / \mathrm{L}$ ). 嗍酸酯与过氧 化氢的反应触发了探针 17 的苂光响应, 释放出具有线 粒体靶向能力的苂光团 17- $\mathrm{NH}_{2}$ (Eq. 2), 并导致 ICT 效 应, 引发了发射光谱中的蓝移现象. 用探针 17 与线粒体 特异性染料罗丹明 123 进行共定位实验, 线粒体中二者 的苂光图像表现出良好的覆盖, 验证了探针 17 可以定 位于线粒体. 比率型荧光探针 $\mathbf{1 7}$ 能准确地反映缺血/再 灌注过程中细胞内源性过氧化氢浓度的变化.

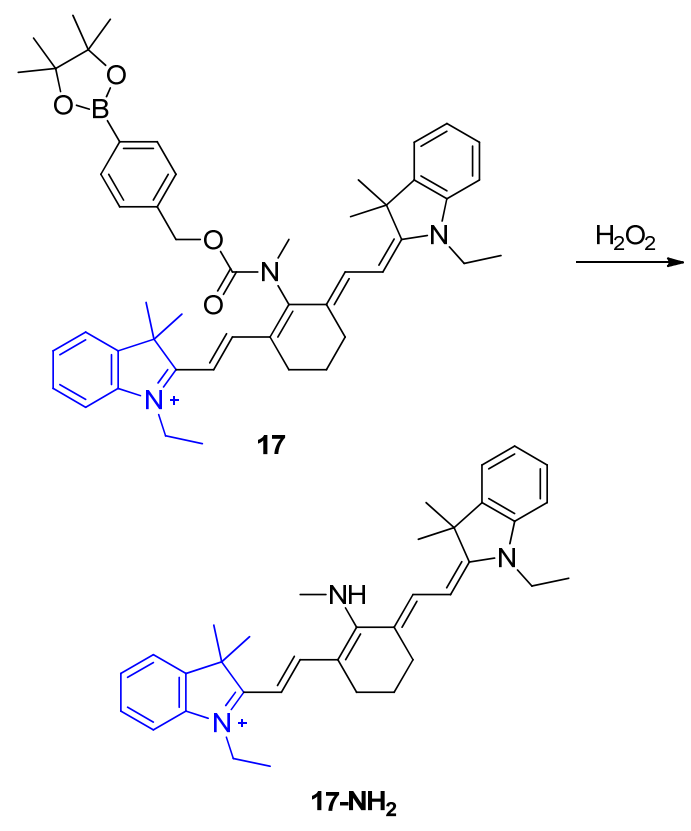

唐波课题组 ${ }^{[51]}$ 还开发了基于吲梠鎓盐结构的线粒 体靶向过氧化氢探针 18. 与过氧化氢反应后, 硼酸酯基 团转化为氧负离子, 形成强烈的推一拉共轭体系, 发生 ICT 效应. 与过氧化氢作用后, 探针 $\mathbf{1 8}$ 在 $558 \mathrm{~nm}$ 处的 荧光强度显著增加, 荧光量子产率从 0.043 增加到 0.15 . 此外, 探针 18 对过氧化氢的检测具有良好的选择性和 较高的灵敏度, 检测限为 $18 \mathrm{nmol} / \mathrm{L}$. 使用探针 $\mathbf{1 8}$ 和线 粒体染料 Mito-Tracker Deep Red 在 HepG2 细胞中进行 共定位实验, 探针与 Mito-Tracker Deep Red 的苂光图像 吻合良好, 共定位系数为 0.95 . 此外, 唐波等 ${ }^{[51]}$ 还合成 了一种新型 $\mathrm{Zn}^{2+}$ 荧光探针 $\left(\lambda_{\mathrm{em}}=779 \mathrm{~nm}\right)$, 用于活细胞和 斑马鱼线粒体内的 $\mathrm{Zn}^{2+}$ 的荧光成像. 同时使用这两个 探针进行双色苂光成像, 发现外源性和内源性过氧化氢 可以刺激线粒体内游离的 $\mathrm{Zn}^{2+}$ 浓度的增加, 这是首次 表明线粒体过氧化氢和 $\mathrm{Zn}^{2+}$ 的协同变化.

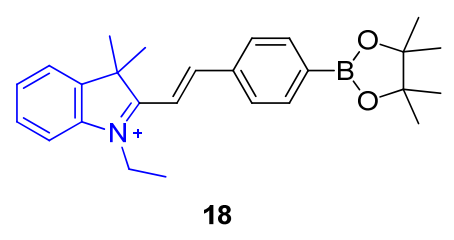

以吲哚鎓盐作为靶向位点的过氧化氢探针, 经实验 证明可以特异性定位于线粒体, 并已被成功应用于细胞 和生物体的荧光成像. 本文中列举的吲哚阳离子探针基 于 ICT 机制, 探针的苂光量子产率通常较低, 今后吲哚 阳离子探针的设计可以在此基础上进行改进.

\section{5 其他阳离子型探针}

2019 年, 周礼义课题组 ${ }^{[52]}$ 报道了一个新的双光子 近红外发射苂光探针 19 , 它以氧鎓离子为线粒体靶向 基团, 以苯硼酸为过氧化氢的传感基团和 ICT 过程的掩 蔽基团. 该探针可在红色荧光通道中 $170 \mu \mathrm{m}$ 深度的肝 组织中进行过氧化氢成像. 由于硼酸单元对 ICT 效应的 限制, 探针 19 的荧光强度被部分猝灭. 加入过氧化氢 后, 硼酸部分经氧化-水解变成差基, 从而恢复 ICT 效 应. 与过氧化氢反应后, 探针 19 在 $665 \mathrm{~nm}$ 处的荧光强 度增加了 105 倍以上. 用探针 19 和罗丹明 123 共同孵育 HeLa 细胞, 然后用过氧化氢处理, 探针 19 的荧光强度 明显增强, 并且与罗丹明 123 通道的成像重叠良好. 此 外，探针 19 能够以单光子和双光子两种方式对线粒体 过氧化氢进行成像.

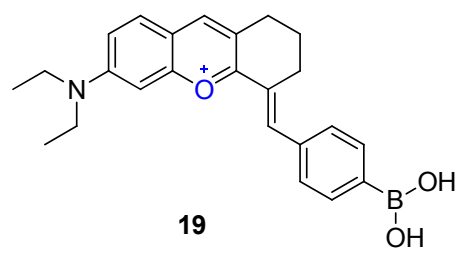

2020 年, 胡利明课题组 ${ }^{[53]}$ 合成了一种结构新颖的 罗丹明衍生物苂光探针 20 , 它以 4 -硝基- $\alpha$-酮酰胺基团 作为过氧化氢的识别基团, 用于检测活细胞中过氧化氢 的水平变化. 结果表明, 该探针的斯托克斯位移为 140 $\mathrm{nm}$, 远大于其他报道的罗丹明衍生物的斯托克斯位移. 由于 4-硝基- $\alpha$-酮酰胺引入了供体激发光诱导电子转移 ( $d$-PET) 过程, 探针 20 的荧光强度非常弱. 与过氧化氢 反应后，4-硝基- $\alpha$-酮酰胺基团被裂解，释放出一个新的 荧光团 20-NH 2 (Eq. 3), 并在 $730 \mathrm{~nm}$ 处产生非常强的荧 光. 此外, 探针对过氧化氢具有出色的选择性和灵敏度, 检测限为 $61 \mathrm{nmol} / \mathrm{L}$. 使用探针 $\mathbf{2 0}$ 和罗丹明 123 进行细 胞共定位实验, 二者的苂光光谱有很好的重叠, 皮尔森 共定位系数为 0.91 , 显示该探针具有靶向线粒体的能 力. 重要的是, 探针 $\mathbf{2 0}$ 可以直观检测活细胞中内源性过 
氧化氢, 还能选择性靶向 HeLa 细胞的线粒体. 多数罗 丹明衍生物的斯托克斯位移小于 $50 \mathrm{~nm}$, 可能会导致苂 光检测中严重的背景干扰, 而探针 20 具有较大的斯托 克斯位移, 这为罗丹明衍生物的结构设计提供了新思 路.

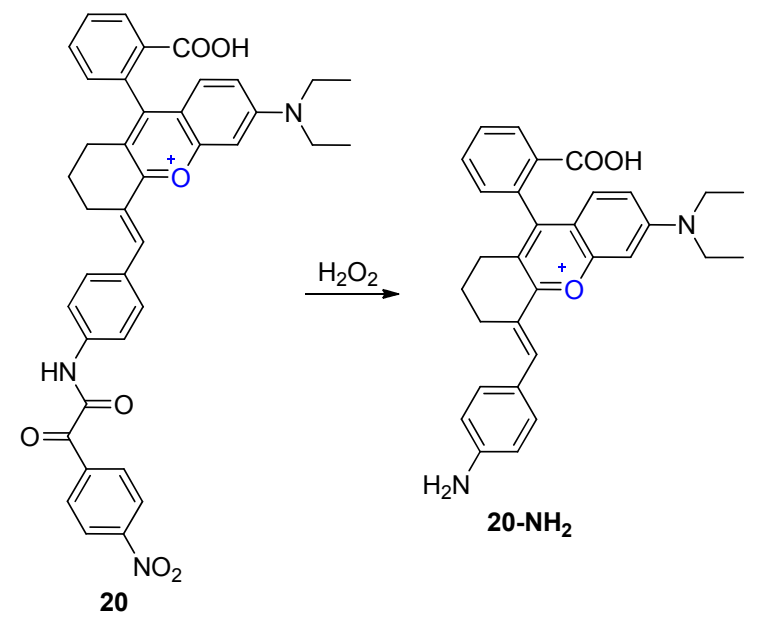

\section{6 总结与展望}

总结了靶向定位于线粒体的过氧化氢荧光探针的 研究进展, 并分别介绍了它们的靶向定位基团、苂光行 为、在生物成像中的应用以及这些荧光传感器的响应机 制. 综上可见，线粒体靶向过氧化氢荧光探针的设计取 得了巨大的进展, 对于许多疾病的预防、诊断以及病理 的研究具有十分重要的意义, 有助于更好地了解亚细胞 器中过氧化氢水平在生理和病理过程中的影响.

然而, 一些线粒体靶向过氧化氢苂光探针的设计仍 需要继续完善: (1)多数已报道的线粒体靶向过氧化氢苂 光探针的发射波长仍然偏短，具有近红外发射波长或红 外发射波长的探针可以实现更深的组织穿透和较低的 背景苂光干扰，还能提高成像的信噪比和灵敏度; (2)大 多数已报道的线粒体靶向过氧化氢荧光探针的响应是 基于 PET、ICT、 TICT 和 FRET 等机制, 可能存在苂光 量子产率低的问题; (3)线粒体靶向过氧化氢苂光探针在 监测或可视化特定细胞器中过氧化氢的水平变化时, 需 要高特异性和耐光性. 但是有些探针在连续的激光照射 下光稳定性较差, 苂光分子聚集时引发猝灭效应; (4)线 粒体靶向过氧化氢荧光探针通常应用于细胞和生物体 中, 对测试条件中的水含量有一定的要求. 目前报道的 一些探针难以在高水含量的条件下进行测试. 基于聚集 诱导发光(AIE)机制的苂光探针具有很多优势, 如大的 斯托克斯位移、较高的苂光量子产率以及在水溶液中实 现对被分析物的检测等, 因此可以将其引入线粒体靶向 过氧化氢苂光探针的设计中，有望解决上述问题.
我们坚信，线粒体靶向过氧化氢荧光探针的研究将 会取得更大的进展，并成为化学家、生物学家和医学家 进行疾病诊断、生理和病理机制研究的强大分子工具.

\section{References}

[1] Finkel, T. Curr. Opin. Cell Biol. 2003, 15, 247.

[2] Stone, J. R.; Yang, S. Antioxid. Redox Signaling 2006, 8, 243.

[3] Zhang, S.; Feng, T.-T.; Zhang, L.; Zhang, M.-N. Chin. J. Anal. Chem. 2019, 47, 1664 (in Chinese). (张帅, 冯涛涛, 张丽, 张美宁, 分析化学, 2019, 47, 1664.)

[4] Reth, M. Nat. Immunol. 2002, 3, 1129.

[5] Dickinson, B. C.; Chang, C. J. Nat. Chem. Biol. 2011, 7, 504.

[6] Veal, E.; Day, A. Antioxid. Redox Signaling 2011, 15, 147.

[7] Levitan, I.; Volkov, S.; Subbaiah, P. V. Antioxid. Redox Signaling 2010, 13, 39.

[8] Brieger, K.; Schiavone, S.; Miller, F. J.; Krause, K. H. Swiss Med. Wkly. 2012, 142, w13659.

[9] Zheng, D.-J.; Yang, Y.-S.; Zhu, H.-L. TrAC, Trends Anal. Chem. 2019, 118, 625.

[10] Zhang, Y.; Dai, M.; Yuan, Z. Anal. Methods 2018, 10, 4625.

[11] Tang, L.; Xia, J.; Zhong, K.; Tang, Y.; Gao, X.; Li, J. Dyes Pigm. 2020, 178,108379

[12] Wen, Y.; Huo, F.; Yin, C. Chin. Chem. Lett. 2019, 30, 1834.

[13] Zhang, J.-D.; Liu, H.-Z.; Meng, L. Chin. J. Org. Chem. 2019, 39, 3132 (in Chinese) (张继东, 刘鸿泽, 孟丽, 有机化学, 2019, 39, 3132.)

[14] Tang, L.; Zhou, L.; Yan, X.; Zhong, K.; Gao, X.; Li, J. J. Photochem. Photobiol., A 2020, 387, 112160.

[15] Yuan, L.; Lin, W.; Zheng, K.; He, L.; Huang, W. Chem. Soc. Rev. 2012, 42,622 .

[16] Chan, J.; Dodani, S. C.; Chang, C. J. Nat. Chem. 2012, 4, 973.

[17] Chen, X.; Pradhan, T.; Wang, F.; Kim, J. S.; Yoon, J. Chem. Rev. 2012, 112, 1910.

[18] Zhang, S.-X.; Niu, Q.-M.; Wu, S.-Z.; Lv, H.-J.; Xing, G.-W. Chin. J. Org. Chem. 2019, 39, 940 (in Chinese). (张晟曦，牛晴旻，吴松泽，吕海娟，邢国文，有机化学，2019, 39, 940.)

[19] Chen, K.; Han, B.-C.; Ji, S.-X.; Sun, J.; Gao, Z.-Z.; Hou, X.-F. Acta Chim. Sinica 2019, 77, 365 (in Chinese). (陈凯，韩百川，嵇思金金，孙瑾，高振忠，侯贤锋，化学学报， 2019, 77, 365.)

[20] Banh, S.; Treberg, J. R. FEBS Lett. 2013, 587, 1799.

[21] Murphy, M. P. Biochem. J. 2009, 417, 1.

[22] Dickinson, B. C.; Srkun, D.; Chang, C. J. Curr. Opin. Chem. Biol. 2010, 14,50

[23] Bao, L.; Avshalumov, M. V.; Patel, J. C.; Lee, C. R.; Miller, E. W.; Chang, C. J.; Rice, M. E. J. Neurosci. 2009, 29, 9002.

[24] Yang, Q.; Wang, S.; Li, D.; Yuan, J.; Xu, J.; Shao, S. Anal. Chim. Acta 2020, 1103, 202.

[25] Zhang, R.; Niu, G.; Liu, Z.; Chau, J. H. C.; Su, H.; Lee, M. M. S.; Gu, Y.; Kwok, R. T. K.; Lam, J. W. Y.; Tang, B. Z. Biomaterials 2020, 242, 119924

[26] Yang, Z.-G.; Xiong, J.; Zhang, W.; Li, W.; Pan, W.-H.; Zhang, J.-G.; Gu, Z.-Y.; Huang, M.-N.; Qu, J.-L. Acta Chim. Sinica 2020, 78, 130 (in Chinese) (杨志刚，熊佳，张炜，李文，潘文慧，张建国，顾振宇，黄美娜， 屈军乐, 化学学报, 2020, 78, 130.)

[27] Horobin, R.; Rashiddoubell, F.; Pediani, J.; Milligan, G. Biotech. Histochem. 2013, 88, 440.

[28] Modicanapolitano, J. S.; Aprille, J. R. Adv. Drug Delivery Rev. 2001, 49, 63

[29] He, L.; Liu, X.; Zhang, Y.; Yang, L.; Fang, Q.; Geng, Y.; Chen, W.; Song, X. Sens. Actuators, B 2018, 276, 247.

[30] Murphy, M. P.; Smith, R. A. J. Annu. Rev. Pharmacol. Toxicol. 2007, 47, 629

[31] Yousif, L. F.; Stewart, K. M.; Kelley, S. O. ChemBioChem 2009, 
10, 1939.

[32] Hardy, M.; Chalier, F.; Ouari, O.; Finet, J. P.; Rockenbauer, A.; Kalyanaraman, B.; Tordo, P. Chem. Commun. 2007, 1083.

[33] Dickinson, B. C.; Chang, C. J. J. Am. Chem. Soc. 2008, 130, 9638.

[34] Masanta, G.; Heo, C. H.; Lim, C. S.; Bae, S. K.; Cho, B. R.; Kim, H. M. Chem. Commun. 2012, 48, 3518.

[35] Du, F.; Min, Y.; Zeng, F.; Yu, C.; Wu, S. Small 2014, 10, 964.

[36] Xu, K.; Qiang, M.; Gao, W.; Su, R.; Li, N.; Gao, Y.; Xie, Y.; Kong, F.; Tang, B. Chem. Sci. 2013, 4, 1079.

[37] Xiao, Y.-F. M.S. Thesis, East China Normal University, Shanghai, 2016 (in Chinese). (肖瑜峰, 硕士论文, 华东师范大学, 上海, 2016.)

[38] Liu, J.; Liang, J.; Wu, C.; Zhao, Y. Anal. Chem. 2019, 91, 6902.

[39] Xu, J.; Zhang, Y.; Yu, H.; Gao, X.; Shao, S. Anal. Chem. 2016, 88 , 1455.

[40] Roopa; Kumar, N.; Bhalla, V.; Kumar, M. Chem. Commun. 2015, $51,15614$.

[41] Ren, M.; Deng, B.; Zhou, K.; Kong, X.; Wang, J.; Lin, W. Anal. Chem. 2016, 89, 552.

[42] Liu, Y.; Niu, J.; Nie, J.; Meng, F.; Lin, W. New J. Chem. 2017, 41, 3320.
[43] Tang, L.; Tian, M.; Chen, H.; Yan, X.; Zhong, K.; Bian, Y. Dyes Pigm. 2018, 158, 482.

[44] Yang, L.; Niu, J.; Sun, R.; Xu, Y.; Ge, J. Analyst 2018, 143, 1813.

[45] Li, H.; Xin, C.; Zhang, G.; Han, X.; Qin, W.; Zhang, C.; Yu, C.; Jing, S.; Li, L.; Huang, W. J. Mater. Chem. B 2019, 7, 4243.

[46] Wu, Z.; Liu, M.; Liu, Z.; Tian, Y. J. Am. Chem. Soc. 2020, 142, 7532.

[47] Li, S.; Wang, P.; Feng, W.; Xiang, Y.; Dou, K.; Liu, Z. Chem. Commun. 2020, 56, 1050.

[48] Xiao, H.; Li, P.; Hu, X.; Shi, X.; Zhang, W.; Tang, B. Chem. Sci. 2016, 7, 6153

[49] Xie, X.; Yang, X.; Wu, T.; Li, Y.; Li, M.; Tan, Q.; Wang, X.; Tang, B. Anal. Chem. 2016, $88,8019$.

[50] Xu, R.; Wang, Y.; You, H.; Zhang, L.; Wang, Y.; Chen, L. Analyst 2019, 144, 2556

[51] Xiao, H.; Li, P.; Zhang, S.; Zhang, W.; Zhang, W.; Tang, B. Chem. Commun. 2016, 52, 12741.

[52] Zhou, L.; Ding, H.; Zhao, W.; Hu, S. Spectrochim. Acta, Part A 2019, 206, 529.

[53] Gu, T.; Mo, S.; Mu, Y.; Huang, X.; Hu, L. Sens. Actuators, B 2020 , 309,127731

(Cheng, F.) 